\title{
Discussion of the First Four Presentations
}

M. Tso: The first comment I would like to make is that the word 'choriocapillaris' is a misnomer. The choriocapillaris is basically a sinusoid and is not comparable to the regular capillaries elsewhere as in the brain or retina. And it is because of its unique sinusoidal characteristics that it is so difficult to study with the conventional cross-sectional technique. This is why I would like to congratulate Dr. Shimizu for showing his elegant technique of studying the choriocapillaris in three dimensions. I would like to address a question to Dr. Shimizu as well as to the rest of the audience. Has anybody been able to use this technique on the human pathology?

As to the controversy between Dr. Hayreh and Dr. Shimizu I wonder whether they would accept that the differences in their interpretations may be due to the fact that the choriocapillaris in the human and in the primates is a basically inhomogeneous structure. At the posterior pole, it is very segmental, while out at the equator, or in the periphery, the segmental pattern becomes less obvious. I wonder whether they would accept this as a possible explanation for the difference in their interpretation of their findings.

Finally I would like to ask Dr. Amalric one question. Namely, what is choroidal circulation given for? This may be a more fundamental question to ask because we know that the choroidal tissue has the highest blood flow per weight of tissue in the body, and recent work shows that the choriocapillaris has a very low oxygen extraction rate, in spite of its fast flow. Maybe Dr. Amalric can enlighten us on the primary question of why we need the choriocapillaris at all. K. Shimizu: All my work has been done on monkey eyes and it has not been possible to do it in removed human eyes. This would be interesting but it is technically very difficult. The second question was the difference between Dr. Hayreh's and my opinion. I think we are essentially saying the same thing. I said that there are anastomoses between terminal arterioles just before they join the choriocapillaris but we did not find anastomoses between major arteries. We found interarteriolar but no interarterial anastomoses. Furthermore, I admitted that the major veins are of a basically segmental pattern; particularly in the horizontal meridian they are bordered by the long posterior ciliary artery which serves as a watershed zone for the veins. But this does not mean that the macula is more liable to damage in venular insufficiencies because it can be drained in both directions.

S. Hayreh: One has to differentiate between the morbid anatomy and the physiological anatomy. There are structures present which we can demonstrate clearly in the dead eye. But in the living eye they don't seem to be actually working due to some physiological mechanisms. I have no explanation for this. I have seen Drs. Ashton, Ring, Wybar and Fujino, everybody has shown intraocular anastomoses. But yet, when we do fluores-cein studies, we don't find any anastomoses. Similarly, the anterior ciliary arteries and the posterior ciliary arteries have been shown to have anastomoses in the region of the equator. And yet, when you cut the posterior ciliary arteries, the choroid never fills from the front. So, the equatorial region is acting once again like a watershed, though anatomically you can demonstrate anastomoses. So you have to 
take with a pinch of salt what you see with venous cast preparations. In the living eye things are somewhat different and what matters for the understanding of any pathological lesion is what Macular Choriocapillaris Pathology

33

is going on in the living eye. And this is where fluores-cein angiography has really revolutionized our thoughts on the choroid and the understanding of the pathoge-netic mechanisms.

A. Bird: The results of the studies make it possible, conceptually, to see how you could get infarction of the choroid and pigment epithelium. A lot of people accept that placoid and placoidlike disease may be due to ischemia, and it has been suggested that the level of obstruction is at the precapillary arteriole. Now I wonder whether Dr. Shimizu could tell us whether this view is compatible with his anatomical studies?

S. K. Shimizu: From what I have found, it appears that the submacular choroid shows a basically segmen-tal arrangement, but for one unit of choriocapillaris you have more than one feeding arteriole; because of this peculiar vascular supply, several arterioles have to be occluded simultaneously.

A. Bird: So you would conclude that to get focal infarcts you need a diffuse vascular abnormality in the choriocapillaris - that is, that many arterioles would have to be influenced to achieve focal infarction. I think that is very much compatible with clinical studies where we see abnormal choriocapillaris filling throughout the posterior pole of an eye in which there are very few apparent infarcts. And interestingly, too, we have one or two patients with unilateral disease but angiography of the apparently normal eye also shows abnormal choroidal transit patterns, suggesting that you can have a bilateral disorder but only unilateral disease.

$\mathrm{D}$, Gass: It seems to me that all evidence suggests that the choriocapillaris is a sinusoidal network; there is relative free communication from the endless sinusoidal network which is being fed by multiple arterioles and drained by multiple venules. The fact that rapidly taken photographs of fluorescein, injected at a higher speed into these arteries, show a segmental pattern tells nothing about the function of the choriocapillaris in the living human. And I would second what Dr. Shimizu said. I cannot visualize that the occlusion of one vertical and one precapillary arteriole would do anything in the eye, because the blood flow would simply shift from the

capillary bed and the adjacent lobule to the obstructed one. So I think that Dr. Hayreh is trying to make too much in interpreting his angiograms and I think that you cannot ignore the fact that from the anatomical point of view, there is free communication in the choriocapillaris in the eye. S. Hayreh: As regards Dr. Gass's comments, this is something which I have seen consistently and it does happen. The angiograms shown here were done on monkeys, but I think you would have similar findings in humans. In young patients you can always find a similar staining. In the older patient, the dye comes so slowly that you cannot really define the border. If you really want to define the choriocapillaris in the human, have a look at patients with ocular hypertension. In these patients you can define the staining pattern precisely because once you slow down the choroidal circulation it becomes easy to see the various lobules. Normally, the whole choroid fills so rapidly that ordinary fluorescein angiography, which takes pictures every $0.8 \mathrm{~s}$, is too slow to show the different phases.

The fact that we do have various localized lesions once again corroborates what we are seeing as the lobu-lized nature of the choriocapillaris. If the filling were to take place from all sides and the 
whole network were communicating then it would be difficult to explain the localized nature of lesions as, for example, in placoid pigment epitheliopathy and other lesions. So, I think it is very easy to say that these are findings on monkeys and just to ignore them. I do not think that it is really true because they do simulate the human in every other way.

I. /. Cunha- Vas: I would like to ask a question to Dr. Singh Hayreh. Have you done during your experimental studies any fluorescein-angiographic studies after experimental denervation? It may be that the differences observed in in vivo and in postmortem studies can be explained on the basis of vascular tonus and the rich vascular innervation of the choroid.

S. Hayreh: No these are perfectly normal eyes. I have not done anything to the ciliary nerve supply. 